\title{
Blasts Under 10,000 of Peripheral Blood White Cells
}

National Cancer Institute

\section{Source}

National Cancer Institute. Blasts Under 10,000 of Peripheral Blood White Cells. NCI

Thesaurus. Code C154096.

A quantitative microscopic finding indicating that less than 10,000 of the total number of nucleated cells in a peripheral leukocyte sample are immature mononuclear cells. 